\title{
A CHARACTERIZATION OF THE GROUP OF HOMOGRAPHIC TRANSFORMATIONS
}

\author{
P. O. BELL
}

1. Introduction. The objectives of this note are three-fold: (1) to present a new differential geometric characterization of the group of homographic transformations of a complex variable, (2) to interpret in geometrical language the significance of the invariance of the Schwarzian derivative under a homographic transformation, and (3) to characterize a general homographic transformation by its unique association with two families of concentric circles.

2. Preliminaries. Let the equation

$$
w=w(z)
$$

denote a conformal representation of the points $z=x+i y$ of a region $R$ of the $z$-plane on the points $w=u+i v$ of a region $\bar{R}$ of the $w$-plane, whereby a general curve $C$ is transformed into a curve $\bar{C}$. Let $\gamma$ and $\bar{\gamma}$ denote the curvatures of $C$ and $\bar{C}$ at corresponding points $z$ and $w$, and let $s$ and $\bar{s}$ denote corresponding lengths of arc of $C$ and $\bar{C}$. For a given transformation (1) it is well known that the rate of variation $d s / d \bar{s}$ is a function $\lambda(x, y)$ which may be expressed in any one of the following forms $\left(u_{x}^{2}+u_{y}^{2}\right)^{-1 / 2},\left(u_{x}^{2}+v_{x}^{2}\right)^{-1 / 2},\left(u_{y}^{2}+v_{y}^{2}\right)^{-1 / 2},\left(v_{x}^{2}+v_{y}^{2}\right)^{-1 / 2}$.

Comenetz ${ }^{1}$ (using a different notation) has obtained, by elementary methods, the formula

$$
\bar{\gamma}=\gamma \lambda+\lambda_{y} \cos \theta-\lambda_{x} \sin \theta,
$$

wherein $\theta=\arctan (d y / d x)$, which is the law of transformation of curvature in conformal mapping, and the formula

$$
d \bar{\gamma} / d \bar{s}=\lambda^{2} d \gamma / d s+\lambda\left[\lambda_{x y} \cos 2 \theta+\frac{1}{2}\left(\lambda_{y y}-\lambda_{x x}\right) \sin 2 \theta\right],
$$

which is the law of transformation of the rate of change of curvature with respect to arc length, under conformal mapping.

3. A new characterization of the group of homographic transformations. It is known that the most general directly conformal transformation which carries circles into circles (including straight lines) is a homographic transformation. The transformation (1) will be such a transformation if and only if

${ }^{1}$ Comenetz, Kasner's invariant and trihornometry, The American Mathematical Monthly, vol. 45 (1938), pp. 82-87. 


$$
\lambda_{x x} \equiv \lambda_{y y}, \quad \lambda_{x y} \equiv 0 .
$$

For if these relations hold, it follows from (3) that

$$
d \bar{\gamma} / d \bar{s}=\lambda^{2} d \gamma / d s .
$$

Hence circles are transformed into circles. Conversely, if circles are transformed by (1) into circles, we must have at corresponding points of an arbitrarily selected circle $C$ and its correspondent $\bar{C}$

$$
d \bar{\gamma} / d \bar{s}=\lambda^{2} d \gamma / d s=0 .
$$

Equation (3) also holds. Hence, no matter what the direction of $C$ at $z$ may be, the following equation must hold:

$$
\lambda\left(\lambda_{y y}-\lambda_{x x}\right)(\sin 2 \theta) / 2+\lambda \lambda_{x y} \cos 2 \theta=0 .
$$

This equation must, therefore, be satisfied independently of $\theta$, and conditions (4) necessarily follow. We may state, therefore, the following theorem.

THeORem 1. A necessary and sufficient condition that a confarmal transformation be a homographic transformation is that the associated function $\lambda(x, y)$ satisfy both of the following identities

$$
\lambda_{x x}-\lambda_{y y} \equiv 0, \quad \lambda_{x y} \equiv 0 .
$$

A geometric interpretation of this condition is that at a general point w of the curve $\bar{C}$ the rate of variation $d \bar{\gamma} / d \bar{s}$ of the curvature of $\bar{C}$ per unit length of arc $\bar{s}$ is independent of the direction of the curve $C$ at the corresponding point $z$.

Equation (3) shows that this is equivalent to the following characterization.

THEOREM 2. The group of homographic transformations consists of all of the conformal transformations under which the differential form $d \gamma d s$ is absolutely invariant.

The following theorem may be deduced, similarly, in consideration of equation (2).

THEOREM 3. A necessary and sufficient condition that a transformation (1) be a directly conformal collineation is that the associated function $\lambda(x, y)$ satisfy both of the identities

$$
\lambda_{x} \equiv 0, \quad \lambda_{y} \equiv 0 .
$$

Under this condition the differential form $\gamma d s$ is an absolute invariant of the transformation (1). 
Since $\gamma=d \theta / d s$ where $d z / d s=e^{i \theta}$, the differential form $d \gamma d s$ may be written in the form

$$
\left(d^{2} \theta / d s^{2}\right)(d s)^{2}
$$

wherein $\theta=-i \log (d z / d s)$.

4. The Schwarzian derivative. Consider a curve defined by

$$
z=x(t)+i y(t)
$$

wherein $x$ and $y$ are functions of a real variable $t$. It is known that the Schwarzian derivative

$$
\{z, t\} \equiv\left(d^{3} z / d t^{3}\right) /(d z / d t)-\frac{3}{2}\left[\left(d^{2} z / d t^{2}\right) /(d z / d t)\right]^{2}
$$

is an absolute invariant under the homographic transformations. Let us investigate the geometric significance of the invariance of this derivative.

We find that

$$
z^{\prime \prime} / z^{\prime}=i \gamma
$$

where accents indicate differentiation with respect to $s$. On differentiating the members of equation (7) with respect to $s$ we obtain

$$
z^{\prime \prime \prime} / z^{\prime}-\left(z^{\prime \prime} / z^{\prime}\right)^{2} \equiv i d \gamma / d s .
$$

Making use of (7) and (8) we deduce

$$
\{z, s\} \equiv i d \gamma / d s+\gamma^{2} / 2 \text {. }
$$

If we make a change of variable by the formula ${ }^{2}$

$$
\{z, t\} \equiv\{z, s\}(d s / d t)^{2}+\{s, t\},
$$

we obtain

$$
\{z, t\} \equiv\left(i d \gamma / d s+\gamma^{2} / 2\right)(d s / d t)^{2}+\{s, t\} .
$$

The real and imaginary components of $\{z, t\}$,

$$
R \equiv\left(\gamma^{2} / 2\right)(d s / d t)^{2}+\{s, t\}, \quad I \equiv(d \gamma / d s)(d s / d t)^{2},
$$

are, themselves, absolute invariants of the group of homographic transformations. Thus, if (1) is homographic, we have

$$
\begin{aligned}
\left(\gamma^{2} / 2\right)(d s / d t)^{2}+\{s, t\} & \equiv\left(\bar{\gamma}^{2} / 2\right)(d \bar{s} / d t)^{2}+\{\bar{s}, t\}, \\
(d \gamma / d s)(d s / d t)^{2} & \equiv(d \bar{\gamma} / d \bar{s})(d \bar{s} / d t)^{2} .
\end{aligned}
$$

\footnotetext{
2 For the change of variable formula see, for example, Ford, Automorphic Func
} tions, McGraw-Hill, 1929, p. 99. 
If now we put $t=s$ in (12), we find

$$
2\{\bar{s}, s\} \equiv \gamma^{2}-\bar{\gamma}^{2}(d \bar{s} / d s)^{2} .
$$

Likewise equation (12) yields

$$
2\{s, \bar{s}\} \equiv \bar{\gamma}^{2}-\gamma^{2}(d s / d \bar{s})^{2}
$$

on substituting $\bar{s}$ for $t$.

Equation (13) expresses the invariance of the form $d \gamma d s$. Making use of this equation and equations (14) and (15), we deduce

$$
\begin{aligned}
& 2\{\bar{s}, s\} \equiv \gamma^{2}-\bar{\gamma}^{2}(d \gamma / d \bar{\gamma})^{2}, \\
& 2\{s, \bar{s}\} \equiv \bar{\gamma}^{2}-\gamma^{2}(d \bar{\gamma} / d \gamma)^{2} .
\end{aligned}
$$

These equations express the significance of the invariance of the Schwarzian derivatives $\{z, s\}$ and $\{z, \bar{s}\}$ as intrinsic geometric relations between any pair of curves $C, \bar{C}$ which correspond under a homographic transformation. To complete the geometric interpretations of (16) and (17) let us recall the significance of the Schwarzian derivative of a real function. ${ }^{3}$ Consider two real functions $\sigma=\sigma(s)$ and $\bar{\sigma}=\bar{\sigma}(s)$ which are chosen to satisfy

$$
\{\sigma, s\} \equiv\{\bar{\sigma}, s\}
$$

identically in $s$. This relation is necessary and sufficient that $\sigma(s)$ and $\bar{\sigma}(s)$ be connected by a homographic transformation

$$
\bar{\sigma}(s)=[a \sigma(s)+b] /[c \sigma(s)+d],
$$

wherein $a, b, c, d$ are constants and $a d-b c \neq 0$. The relation (18) is also necessary and sufficient that corresponding to any set of four values $s=s_{j},(j=1,2,3,4)$, the cross-ratios

$$
\left(\sigma_{1}, \sigma_{2}, \sigma_{3}, \sigma_{4}\right), \quad\left(\bar{\sigma}_{1}, \bar{\sigma}_{2}, \bar{\sigma}_{3}, \bar{\sigma}_{4}\right)
$$

are identical. If as $s$ varies, points $P$ and $\bar{P}$ describe curves whose corresponding lengths of arc are defined by $\sigma=\sigma(s)$ and $\bar{\sigma}=\bar{\sigma}(s)$, the movements of these points will be called projectively applicable. This designation is suggested by the property that the development of these movements along a straight line produces projectively equivalent rectilinear movements.

Corresponding to a real single-valued differentiable function $\sigma=\sigma(s)$ there exists a class $\mathfrak{S}_{\sigma(s)}$ of projectively applicable movements to which a movement defined by $\bar{\sigma}=\bar{\sigma}(s)$ will be said to belong if $\{\bar{\sigma}, s\} \equiv\{\sigma, s\}$. We shall call the Schwarzian $\{\sigma, s\}$ the absolute projective acceleration

\footnotetext{
${ }^{3}$ Cartan, Leçons sur la Théorie des Espaces, Paris, Gauthier-Villars, 1937, p. 3.
} 
of the movements of the class $\mathfrak{S}_{\sigma(s)}$ or simply the absolute projective acceleration $^{4}\{\sigma, s\}$.

We may now state the following theorem:

THEOREM 4. The invariance of the Schwarzian derivative $\{z, t\}$ yields the identities (16) and (17) which express the absolute projective accelerations $\{\bar{s}, s\}$ and $\{s, \bar{s}\}$ algebraically in terms of the squares of the curvatures $\gamma$ and $\bar{\gamma}$ and the square of their rate of variation $d \gamma / d \bar{\gamma}$.

Other interesting identities may be obtained by forming various combinations of (13), (14) and (15). One of these has the surprisingly simple form

$$
\bar{\gamma}^{2} /\{s, \bar{s}\}+\gamma^{2} /\{\bar{s}, s\} \equiv 2 .
$$

5. The magnimetric circles. Let us consider a homographic transformation

$$
w=(a z+b) /(c z+d),
$$

where $a, b, c, d$ are constants and $a d-b c=1$. Since $\lambda(x, y)=|d z / d w|$ and for (20) $d z / d w$ is defined by $d z / d w=(c z+d)^{2}$, we may write

$$
\lambda(x, y) \equiv(c z+d)(\bar{c} \bar{z}+\bar{d}),
$$

where $\bar{z}, \bar{c}, \bar{d}$ denote the conjugate imaginaries of $z, c, d$. Let the value of $\lambda$ defined at the point $z_{1}=x_{1}+i y_{1}$ be denoted by $\lambda_{1}$. By making use of (20), (21) and the equation

$$
z=(-d w+b) /(c w-a),
$$

for the inverse of (20), the proof of the following theorem may be sup plied by the reader.

TheOREM 5. Through a point $z_{1}$, in the $z$-plane (excluding $z_{1}=\infty$ and $\left.z_{1}=-d / c\right)$, there passes just one circle which by (20) is magnified in all of its elements of arc length by the constant multiple $1 / \lambda_{1}$. Similarly, through the point $w_{1}$, which corresponds by (20) to the point $z_{1}$, there passes just one circle which by (22) is magnified in all of its elements of arclength by the constant multiple $\lambda_{1}$.

These circles will be called magnimetric circles of the transformations (20) and (22). The totality of the magnimetric circles in the z-plane form a family of concentric circles, a general one of which is defined by

$$
(c z+d)(\bar{c} \bar{z}+\bar{d})=\lambda, \quad \lambda=\text { const. }
$$

${ }^{4}$ Loc. cit., Footnote 3, p. 3. Cartan, in considering rectilinear motion defined by $x=x(t)$ has called the Schwarzian $\{x, t\}$ "l'accélération projective du mouvement." 
The corresponding magnimetric circles in the $w$-plane form the family of concentric circles, a general one of which is defined by

$$
(c w-a)(\bar{c} \bar{w}-\bar{a})=1 / \lambda .
$$

Let $r(z)$ and $\rho(w)$ denote the radii of the circles (23) and (24), respectively. We have, clearly, that

$$
r^{2}(z)=\lambda / c \bar{c}, \quad \rho^{2}(w)=1 / \lambda c \bar{c} .
$$

When $\lambda=1$, equations (24) and (25) represent the isometric ${ }^{5}$ circles. We shall refer to families in the $z$ - and $w$-planes as $z$ - and $w$-families, respectively. By making use of equations (23) and (24) in connection with $(20)$, the following theorem is obtained.

THEOREM 6. There are $\infty^{2}$ transformations of the form (20) which transform a z-family of concentric circles with an arbitrarily selected center $z_{0}$ into a w-family of concentric circles with an arbitrarily selected center $w_{0}$. There are $\infty^{1}$ of these which transform a selected circle of the z-family into a selected circle of the w-family. On requiring this pair of circles to correspond, a one-to-one correspondence among the other members of the two families is established. Finally, there is just one transformation of this infinite system which transforms a selected point $z_{1}$ on any circle of the z-family into a selected point $w_{1}$ on the corresponding circle of the w-family. The circles of these z- and w-families are the magnimetric circles of the transformation, and the product of any corresponding pair of radii is equal to $1 /|c|^{2}$.

UNIVERSITY OF KANSAS

${ }^{5}$ Loc. cit., Footnote 2, pp. 23-27. 